\title{
O ENVOLVIMENTO PATERNO E O DESENVOLVIMENTO SOCIAL DE CRIANÇAS INICIANDO AS ATIVIDADES ESCOLARES ${ }^{1}$
}

\author{
Fabiana Cia* \\ Elizabeth Joan Barham ${ }^{\#}$
}

\begin{abstract}
RESUMO. Este estudo relacionou indicadores do envolvimento paterno com indicadores de desenvolvimento social dos filhos. Participaram 97 pares de pais e mães (com filhos na $1^{-}$ou $2^{-}-$série) e 20 professoras. Para avaliar o envolvimento paterno, pais e mães responderam à Avaliação do bem-estar pessoal e familiar e do relacionamento pai-filho - Versão Paterna, e para avaliar o desenvolvimento social das crianças, os pais, as mães e as professoras preencheram o Social Skills Rating System-SSRS. Quanto maior a freqüência de comunicação entre pai e filho e de participação do pai nos cuidados e nas atividades escolares, culturais e de lazer do filho, menor o índice de hiperatividade e de problemas de comportamento e mais adequado o repertório de habilidades sociais das crianças. Os resultados indicam a provável importância do envolvimento positivo do pai para o desenvolvimento social dos filhos e os prováveis benefícios de programas para promover envolvimento paterno.
\end{abstract}

Palavras-chave: envolvimento paterno, habilidades sociais, problemas de comportamento.

\section{FATHER INVOLVEMENT AND THE SOCIAL DEVELOPMENT OF CHILDREN IN THE SCHOOL-ENTRY TRANSITION STAGE}

\begin{abstract}
This study aimed to examine the relationships between indicators of parental involvement and their children's social and included 97 father-mother pairs (parents of children in the first or second grade), and 20 teachers. To evaluate father involvement, fathers and mothers completed an Evaluation of personal and family wellbeing and the parent-child relationship - Fathering Version, and to evaluate the children's social development, the mothers, fathers and teachers completed the Social Skills Rating System-SSRS. The results indicate that more frequent: communication between father and child, paternal participation in child caregiving and in the child's school, cultural and leisure activities are each associated with indicators of lower hyperactivity, fewer behavior problems and a wider repertoire of appropriate social skills. These results point to the importance of the constructive involvement of fathers for their children's social development and the likely benefits of programs to promote father involvement.
\end{abstract}

Key words: Father involvement, social skills, behavior problems.

\section{LA PARTICIPACIÓN PATERNA Y EL DESARROLLO SOCIAL DE NIÑOS INICIANDO LAS ACTIVIDADES ESCOLARES}

RESUMEN. En este estudio se han relacionado indicadores de la participación paterna con los de desarrollo social de los hijos y participaron 97 padres y madres (con hijos en el primero o en el segundo grado) y 20 profesoras. Para evaluar la participación paterna, padres y madres respondieron a la Evaluación del bienestar personal y familiar y del reracionamiento padre-hijo - Versión Paterna y para evaluar el desarrollo social de los niños, padres, madres y profesoras completaron el Social Skills Rating System-SSRS. Cuanto mayor es la frecuencia de comunicación entre padre e hijo, de la participación del padre en los cuidados y en las actividades escolares, culturales y de ocio del hijo, menor es el índice de hiperactividad, problemas de comportamiento y mayor es el repertorio de destrezas sociales de los niños. Los resultados indican la probable importancia de la participación positiva del padre para el desarrollo social de los hijos.

Palabras-clave: Participación paterna, destrezas sociales, problemas de comportamiento.

\footnotetext{
Apoio: Fapesp.

* Psicóloga e Doutoranda no Programa de Pós-Graduação em Educação Especial da Universidade Federal de São Carlos-UFSCar.

\# Doutora em Psicologia Social e de Desenvolvimento Aplicado. Professora Titular do Programa de Pós-Graduação em Educação Especial e do Departamento de Psicologia da Universidade Federal de São Carlos-UFSCar.
} 
Nas últimas décadas surgiu um interesse por entender o potencial do pai para promover o desenvolvimento infantil. Segundo o modelo bioecológico de Bronfenbrenner (1999), as interações entre os pais e seus filhos são influenciadas por um complexo conjunto de fatores pessoais e contextuais, envolvendo interações bidirecionais, as quais são afetadas por crenças e práticas nos diferentes ambientes em que os pais e seus filhos transitam. São cada vez mais numerosos os estudos que demonstram a importância do pai para diferentes aspectos do desenvolvimento infantil, mas ainda existem lacunas nestas informações. Neste estudo procura-se ampliar os conhecimentos nesta área, enfocando a relação entre alguns indicadores do envolvimento paterno e várias medidas do desenvolvimento social infantil, num contexto brasileiro.

O maior interesse pelo papel paterno decorre de pesquisas da área de aprendizagem sociocognitiva (como Bandura, 1986) mostrando que os comportamentos sociais são aprendidos primeiramente na família e depois em outros ambientes nos quais a criança convive (escola, igreja, comunidade, etc.). Sendo assim, além da relação mãe-filho, o relacionamento pai-filho também deve exercer uma influência muito significativa no desenvolvimento dos padrões de comportamento da criança, os quais, por sua vez, influenciarão na qualidade das suas relações interpessoais subseqüentes.

Um dos indicadores da qualidade das relações sociais é o repertório de habilidades sociais do indivíduo. O termo habilidades sociais se refere "a diferentes classes e subclasses de comportamentos sociais do repertório de um indivíduo, que contribuem para a competência social, favorecendo um relacionamento produtivo e saudável com as demais pessoas" (Del Prette \& Del Prette, 2005, p. 31). A aprendizagem das habilidades sociais na infância ocorre principalmente por meio de observação, modelação, instrução ou conseqüenciação (Del Prette \& Del Prette, 2005). Assim, os pais e as mães, que estão em interação cotidiana com as crianças, são os indivíduos com maior probabilidade de gerar ou manter os comportamentos problemáticos da criança, em função de informações, crenças e habilidades educativas inadequadas ou do seu estado psicológico, embora não o façam intencionalmente (Caballo \& Simón, 2005; Del Prette \& Del Prette, 2005). Rocha e Brandão (2001) complementam que os pais podem atuar tanto como mantenedores quanto como modificadores dos comportamentos infantis inadequados. $\mathrm{O}$ fato de poderem ser modificadores é que estimula o interesse em avaliar diferentes aspectos do envolvimento paterno, visto que mudanças nos comportamentos dos pais podem reverter em melhorias no comportamento dos filhos.

Em função do potencial do pai para proteger e estimular seu filho, a importância de verificar a qualidade do envolvimento paterno fica ainda maior quando se pensa em crianças vivendo em contextos de vulnerabilidade social. Atzaba-Poria, Pike e DeaterDeckard (2004) realizaram um estudo com 125 famílias de diferentes níveis socioeconômicos, com o objetivo de verificar a influência de múltiplos fatores de risco sobre a existência de problemas de comportamento em crianças escolares. Verificou-se que as crianças com menor desenvolvimento cognitivo cujos pais e mães tenham mostrado comportamentos parentais negativos (menos calorosos, mais rígidos e menos recíprocos na relação com $\mathrm{o}$ filho) apresentaram maior índice de problemas de comportamento internalizantes (retraimento, queixas somáticas, depressão e ansiedade) e externalizantes (delinqüência, agressão). Do lado positivo, a satisfação conjugal e o suporte social percebido por ambos os pais foram aspectos que contribuíram para melhorar a qualidade do relacionamento entre estes e seus filhos.

Outros estudos também mostram uma relação entre várias habilidades ou estilos parentais e diferentes aspectos do desenvolvimento social das crianças. Por exemplo, Eisenberg et al. (2005) realizaram um estudo longitudinal com 186 crianças, seus pais e suas mães. Os participantes foram avaliados por meio de observações e questionários, em três momentos (quando as crianças tinham nove, $11 \mathrm{e}$ 13 anos, respectivamente). Conforme mostram os resultados, os pais e mães das crianças que apresentaram problemas de comportamento externalizantes, nestas três idades, eram menos calorosos e se expressavam positivamente com baixa frequiência. Além disso, seus filhos tinham menos controle emocional e menor persistência nas tarefas, segundo relatos de ambos os pais e dos professores.

A importância da ligação entre a interação paterna e o desenvolvimento social dos seus filhos fica ainda mais evidente diante de vários estudos que apontam para uma correlação negativa entre os déficits em competência social e habilidades sociais com o desempenho acadêmico (Del Prette \& Del Prette, 2005; Dunn, Cheng, O'Connor \& Bridges, 2004) e uma correlação positiva com problemas no desenvolvimento sociemocional (Bolsoni-Silva \& Del Prette, 2002; Cia, Pamplin \& Del Prette, 2006) e de ajustes psicossociais, no decorrer do desenvolvimento 
(Bongers, Koot, Ende \& Verhulst, 2004; Coley, Morris \& Hernandez, 2004; Oliveira et al., 2002).

Outros estudos apontam para ganhos que podem ser obtidos quando a qualidade da relação pai-filho contribui para o bom desenvolvimento social da criança. Por exemplo, melhor desenvoltura social é um fator de proteção para as crianças que estão ingressando em atividades escolares, porque esta fase de transição requer adaptações elaboradas para lidar com demandas novas. Além das mudanças de contextos físicos e sociais, as expectativas dos interlocutores no ambiente escolar são mais exigentes do que no familiar, pois a dependência é menos tolerada, as regras de convívio social são mais complexas e o suporte é menos disponível. Ao ser julgada por outros, a criança se motiva para corresponder às expectativas, muitas vezes conflitantes, da família, da escola e do grupo de companheiros (Marturano, 2004).

Diante dos estudos ligando a maneira como o pai age com o desenvolvimento social dos seus filhos, percebe-se a importância de melhor entender outros aspectos do envolvimento paterno (por exemplo, o que ele faz). Além da importância do estilo do pai para o desenvolvimento social dos filhos, estudos têm evidenciado a relevância da participação paterna na sua educação, pois no atual contexto cultural brasileiro as práticas educativas de ambos os pais em relação a seus filhos estão num processo de transformação, passando de uma postura de rigidez para uma maior permissividade. Esta mudança reflete a redefinição mais ampla no papel do pai, uma vez que uma porcentagem cada vez maior de mulheres está entrando no mercado de trabalho e os homens estão tendo que assumir um envolvimento maior no âmbito familiar (Brandth \& Kvande, 2002; Cabrera, TamesLeMonda, Bradley, Hofferth \& Lamb, 2000; Dessen \& Costa, 2005; Tiedje, 2004). Esta nova divisão de tarefas pode resultar na melhoria nas relações familiares, pois quando ambos os membros do casal entendem a importância das demandas profissionais e familiares, as crianças se beneficiam do maior envolvimento do pai (Oliveira et al., 2002; Weber, Prado, Viezzer \& Brandenburg, 2004).

Considerando que o envolvimento paterno positivo pode influenciar a competência social de seu filho, agindo como um fator de proteção e de maximização do desenvolvimento infantil, e ainda tendo em vista a escassez de estudos focalizando especificamente o envolvimento paterno (o que o pai faz, e não seu estilo parental), este estudo teve por objetivo relacionar indicadores do envolvimento paterno com algumas medidas centrais do desenvolvimento social dos seus filhos - habilidades sociais e problemas de comportamento (externalizantes e internalizantes).

\section{MÉTODO}

\section{Participantes}

Essa pesquisa contou com a participação de 97 casais (pais e mães) de crianças da $1^{-}$e $2^{2^{-}}$séries do Ensino Fundamental (21,2\% das crianças estavam na $1^{\mathrm{a}}$ série e $78,8 \%$ estavam na $2^{\mathrm{a}}$ série do Ensino Fundamental). Dois casais tinham gêmeos, sendo assim, as respostas dos pais foram referentes a 99 crianças (50 do sexo feminino e 49 do sexo masculino). A média de idade dos pais era de 35 anos (variando entre 23 e 58 anos) e a média de idade das mães era de 32 anos (variando entre 20 e 55 anos). Todos os participantes eram casados ou viviam como casados e todos os pais e $97 \%$ das mães exerciam algum tipo de atividade remunerada, fatores que permitiram controlar algumas variáveis que poderiam influir fortemente no envolvimento paterno. Em relação à classe socioeconômica, $7,1 \%$ das famílias eram de classe socioeconômica D, 50,5\% eram de classe socioeconômica C, 35,4\% eram de classe socioeconômica B2 e 7,1\% eram de classe socioeconômica B1 (segundo Critério Brasil, 2006).

Além disso, participaram deste estudo 20 professoras, com média de idade de 40 anos (variando entre 25 e 59 anos), das quais seis lecionavam para a $1^{a}$ série e 14 para a $2^{a}$ série do Ensino Fundamental. Em média, estas professoras contavam 14 anos de exercício do magistério; duas delas ainda estavam cursando o $3^{-}$grau (com formação em pedagogia) e as demais tinham o $3^{-}$grau completo (15 professoras eram formadas em pedagogia, uma em letras, uma em história e uma em pedagogia e letras).

\section{Local de coleta de dados}

A coleta de dados ocorreu em duas escolas municipais e em uma escola estadual, localizadas em um município do Interior do Estado de São Paulo com cerca de 200.000 habitantes.

\section{Instrumentos}

\section{Pais e mães}

Os instrumentos utilizados para a avaliação do bemestar pessoal e familiar e do relacionamento pai-filho foram: 1- Versão paterna (Cia, D’Affonseca \& Barham, 2004), instrumento composto de três escalas tipo Likert que contemplam uma diversidade de indicadores de envolvimento positivo dos pais com os filhos, sendo elas: 
(a) Escala de comunicação (verbal e não-verbal) entre pai e filho - uma escala tipo Likert de 22 itens, com a pontuação dos itens variando entre 0 , 'nunca' a 365 , 'uma vez por dia' ( $\alpha=0,95$ quando o informante foi o pai e $\alpha=$ 0,96 quando foi a mãe); (b) Escala de participação do pai nos cuidados com o filho - uma escala tipo Likert de 15 itens, com a pontuação dos itens variando entre 1, 'nenhuma participação' a 5, 'muita participação' $(\alpha=0,95$ quando o informante foi o pai, e $\alpha=0,95$ quando foi a mãe) e (c) Escala de participação do pai nas atividades escolares, culturais e de lazer do filho - uma escala tipo Likert de 19 itens, com a pontuação dos itens variando entre 0 , 'nunca' a 365, 'uma vez por dia' ( $\alpha=0,96$ quando o informante foi o pai e $\alpha=0,95$ quando foi a mãe); e 2) o Social Skills Rating System - SSRS - Versão para Pais. Esta versão (elaborada por Gresham \& Elliott, 1990, validada para o nosso contexto por Bandeira, Del Prette, Del Prette \& Magalhães, no prelo) do SSRS foi aplicada para avaliar a percepção dos pais quanto ao repertório de habilidades sociais e à existência e intensidade de problemas de comportamento internalizantes e externalizantes nas crianças, e era composta de duas escalas tipo Likert: (a) 38 itens, em que os pais assinalam qual a frequiência (nunca, algumas vezes e muito freqüente) com a qual a criança age de determinadas maneiras, perante diferentes demandas sociais, sendo que esses itens podem ser agrupados em seis fatores: cooperação, asserção positiva, iniciativa/desenvoltura social, asserção de enfrentamento, civilidade e autocontrole e (b) 17 itens em que os pais assinalam qual a frequiência (nunca, algumas vezes e muito freqüente) com a qual a criança apresenta cada uma das três classes de comportamentos problemáticos (hiperatividade, comportamentos problemáticos externalizantes e comportamentos problemáticos internalizantes).

\section{Professores}

O Social Skills Rating System - SSRS - Versão para Professores (elaborado por Gresham \& Elliott, 1990 e validado para o nosso contexto por Bandeira et al., no prelo) foi aplicado para avaliar a percepção das professoras quanto ao repertório de habilidades sociais, à existência e intensidade de problemas de comportamento internalizantes e externalizantes das crianças em contexto de sala de aula. $\mathrm{O}$ instrumento se compõe de duas escalas tipo Likert: (a) 30 itens em que a professora assinala qual a freqüência (nunca, algumas vezes e muito freqüente) com a qual a criança age de determinadas maneiras perante diferentes demandas sociais, sendo que esses itens podem ser agrupados em cinco fatores: responsabilidade/cooperação, asserção, autocontrole, autodefesa e cooperação com pares; e (b) 18 itens em que a professora assinala qual a freqüência (nunca, algumas vezes e muito freqüente) com a qual a criança apresenta cada uma das duas classes de comportamentos problemáticos (externalizantes e internalizantes).

\section{Procedimento de coleta de dados}

Solicitou-se que ambos os pais e as professoras preenchessem o Termo de Consentimento Livre e Esclarecido, concordando em participar na pesquisa. Com os pais foi agendado um horário para preencherem ao instrumento Avaliação do bem-estar pessoal e familiar e do relacionamento pai-filho Versão paterna e o Social Skills Rating System - SSRS - Versão para pais. A pesquisadora orientou os pais a responderem com base em seu relacionamento com o filho, estudante da $1^{\text {a }}$ ou $2^{\mathrm{a}}$ séries do Ensino Fundamental.

Concomitantemente à coleta de dados com ambos os pais, as professoras preencheram o Social Skills Rating System - SSRS - Versão para professores. A pesquisadora forneceu instruções sobre o preenchimento do instrumento e esclareceu as dúvidas das professoras, dirigindo-se à sala de aula de cada uma.

\section{Procedimento de análise de dados}

Os dados quantitativos, obtidos por meio do instrumento Avaliação do bem-estar pessoal $e$ familiar e do relacionamento pai-filho - Versão paterna, foram analisados em termos descritivos, com medidas de tendência central e dispersão. Para verificar a fidedignidade das medidas, no contexto deste estudo foi realizada uma análise de consistência interna (Alpha de Cronbach) da escala como um todo (Cozby, 2002). As pontuações dos dados obtidos no SSRS - Versão para pais e SSRS - Versão para professores foram realizadas com base nos procedimentos apresentados nos respectivos manuais. As relações entre os indicadores do repertório social dos filhos e os do envolvimento parental foram verificadas por meio do teste de correlação de Pearson $(\mathrm{p}<0.05)$. Para comparar as avaliações do envolvimento paterno feitas pelos dois tipos de informante (pais e mães), foi aplicado o teste-t.

\section{RESULTADOS}

\section{Envolvimento paterno}

Ao comparar a opinião dos pais e das mães quanto ao envolvimento paterno por meio do teste- $t$, verificou-se a inexistência de diferenças estatisticamente significativas 
entre os valores médios para cada um dos itens que compõem as três escalas usadas para verificar o envolvimento paterno em três domínios ligados à educação do filho (comunicação entre pai e filho; participação do pai nos cuidados com o filho; participação do pai nas atividades escolares, culturais e de lazer do filho). Por isso, os dados referentes ao envolvimento paterno estão apresentados na Tabela 1 apenas com as médias gerais para cada escala, porém separados por tipo de informante (pais e mães).

Tabela 1. Médias Gerais nas Subescalas de Envolvimento Paterno: Comparação da Opinião dos Pais e Mães

\begin{tabular}{|c|c|c|c|c|c|c|}
\hline \multirow{2}{*}{ Escalas } & \multicolumn{2}{|c|}{ Pais } & \multicolumn{2}{|c|}{ Mães } & \multirow{2}{*}{$\begin{array}{c}\text { teste } \\
t\end{array}$} & \multirow{2}{*}{$\begin{array}{c}p \\
\text { valo }\end{array}$} \\
\hline & Média & D.P. & Média & D.P. & & \\
\hline $\begin{array}{l}\text { Comunicação (verbal e } \\
\text { não verbal) entre pai e } \\
\text { filho }{ }^{1} \text {. }\end{array}$ & 136,3 & 87,5 & 144,9 & 95,5 & 0,66 & 0,51 \\
\hline $\begin{array}{l}\text { Participação do pai nos } \\
\text { cuidados com o filho }{ }^{2} \text {. }\end{array}$ & 3,01 & 0,99 & 3,05 & 0,97 & 0,33 & 0,74 \\
\hline $\begin{array}{l}\text { Participação do pai nas } \\
\text { atividades escolares, } \\
\text { culturais e de lazer do } \\
\text { filho }{ }^{1} \text {. }\end{array}$ & 121,7 & 94,2 & 120,4 & 90,1 & 0,10 & 0,92 \\
\hline $\begin{array}{l}* \mathrm{p}<0,05 \text { significativo } \\
\text { Notas: } \\
\text { 1. } 0=\text { nunca a } 365=\text { todo } \\
2.1=\text { pouca participação }\end{array}$ & $\begin{array}{r}\text { dias } \\
-m u\end{array}$ & & & & & \\
\hline
\end{tabular}

A maioria dos pais e das mães relatou que o pai usava todas as formas de comunicação com os filhos, duas ou três vezes por semana. O tipo de comunicação que os pais referiram usar com maior freqüência com os filhos foi o de manter diálogo com eles. Os pais apontaram uma frequiência maior de os filhos os procurarem para conversar. Além disso, os pais apresentaram uma pontuação medianamente alta no grau de participação nos cuidados com os filhos, sendo que sua maior participação estava na educação escolar e em passear com os filhos (segundo a opinião dos pais e das mães).

Considerando-se a escala de Participação do pai nas atividades escolares, culturais e de lazer dos filhos, pode-se verificar que, segundo a opinião dos pais e das mães, os pais participavam das atividades escolares, culturais e de lazer dos filhos "uma vez por semana" a "duas ou três vezes por semana". As atividades em que os pais participavam com maior freqüência foram as de brincar com o filho, acompanhar o progresso escolar do filho e auxiliá-lo nas lições de casa.

A Tabela 2 mostra os valores médios e os desviospadrão da avaliação dos pais, das mães e das professoras quanto aos problemas de comportamento das crianças.
Tabela 2. Problemas de Comportamento Atribuídos às Crianças, Segundo Avaliação dos Pais, das Mães e das Professoras

\begin{tabular}{lccc}
\hline & Média & D. P. & Escore médio \\
\hline Avaliação dos pais & & & \\
HIP & 04,5 & 3,27 & $2,37-6,93$ \\
CPI & 02,5 & 2,04 & $1,00-4,16$ \\
CPE & 05,4 & 3,44 & $2,26-7,20$ \\
CPT & 12,5 & 07,7 & $6,24-16,06$ \\
Avaliação das mães & & & \\
HIP & 04,7 & 3,42 & $2,37-6,93$ \\
CPI & 02,7 & 2,17 & $0,90-4,16$ \\
CPE & 05,6 & 3,41 & $2,26-7,20$ \\
CPT & 13,0 & 7,72 & $6,24-16,06$ \\
Avaliação das professoras & & & \\
CPI & 03,9 & 3,25 & $1,27-3,17$ \\
CPE & 10,1 & 6,81 & $3,70-8,31$ \\
CPT & 13,4 & 8,42 & $4,39-9,30$ \\
\hline
\end{tabular}

Legenda: HIP: Hiperatividade; CPI: Comportamentos problemáticos internalizantes; CPE: Comportamentos problemáticos externalizantes; CPT: Comportamentos problemáticos total.

Para os pais e para as mães, as crianças apresentaram uma frequiência média de problemas de comportamento, mas segundo as professoras, as crianças estavam com uma freqüência de problemas de comportamento acima da média, considerando-se a amostra de referência (Bandeira et al., no prelo).

O repertório de habilidades sociais das crianças foi avaliado por ambos os pais e pelas professoras, como mostra a Tabela 3 .

Tabela 3. Repertório de Habilidades Sociais das Crianças, por Fator, na Avaliação dos Pais, das Mães e das Professoras

\begin{tabular}{lccc}
\hline Habilidades sociais & \multicolumn{3}{c}{ MédiaD. P. Escore médio } \\
\hline Pais & 11,5 & 4,98 & $7,13-14,99$ \\
F1-Cooperação. & 10,2 & 3,78 & $9,60-14,87$ \\
F2-Asserção positiva. & 09,7 & 4,15 & $7,82-14,02$ \\
F3-Iniciativa/Desenvoltura social. & 09,9 & 3,98 & $8,59-14,35$ \\
F4-Asserção de enfrentamento. & 07,1 & 2,96 & $4,76-9,08$ \\
F5-Civilidade. & 04,6 & 2,30 & $2,06-5,64$ \\
F6-Autocontrole. & 42,4 & 15,2 & $35,08-53,36$ \\
Total & & & \\
Mães & 11,4 & 5,06 & $7,13-14,99$ \\
F1-Cooperação. & 10,2 & 3,78 & $9,60-14,87$ \\
F2-Asserção positiva. & 09,4 & 4,19 & $7,82-14,02$ \\
F3-Iniciativa/Desenvoltura social. & 10,1 & 4,06 & $8,59-14,35$ \\
F4-Asserção de enfrentamento. & 07,1 & 2,98 & $4,76-9,08$ \\
F5-Civilidade. & 04,7 & 2,19 & $2,06-5,64$ \\
F6-Autocontrole. & 38,4 & 14,4 & $35,08-53,36$ \\
Total & & & \\
Professoras & 21,1 & 6,48 & $15,35-28,61$ \\
F1-Responsabilidade/Cooperação. & 11,5 & 3,68 & $6,86-15,22$ \\
F2-Asserção. & 11,5 & 3,81 & $8,34-16,20$ \\
F3-Autocontrole. & 03,4 & 1,52 & $1,67-5,11$ \\
F4-Autodefesa. & 05,1 & 2,06 & $2,61-6,69$ \\
F5-Cooperação com pares. & 39,7 & 11,2 & $28,73-51,83$ \\
\hline Total & &
\end{tabular}


Considerando-se a opinião dos três informantes, as crianças apresentaram um repertório de habilidade sociais dentro da faixa que capta escores médios, segundo a amostra de referência (Bandeira et al., no prelo).

\section{Relação entre os problemas de comportamento e o} envolvimento paterno

A Tabela 4 relaciona os problemas de comportamento e o repertório de habilidades sociais das crianças com as escalas que compõem o envolvimento paterno.

Tabela 4. Correlações (Pearson) Significativas do Envolvimento Paterno com os Problemas de Comportamento e o Repertório de Habilidades Sociais das Crianças

\begin{tabular}{|c|c|c|c|c|c|c|}
\hline & \multicolumn{3}{|c|}{ Opinião dos pais } & \multicolumn{3}{|c|}{ Opinião das mães } \\
\hline & $\begin{array}{l}\text { Comunicação } \\
\text { entre pai e filho }\end{array}$ & $\begin{array}{c}\text { Participação do pai } \\
\text { nos cuidados com o } \\
\text { filho }\end{array}$ & $\begin{array}{l}\text { Participação do pai } \\
\text { nas atividades } \\
\text { escolares, culturais e } \\
\text { de lazer do filho }\end{array}$ & $\begin{array}{l}\text { Comunicação } \\
\text { entre pai e } \\
\text { filho }\end{array}$ & $\begin{array}{l}\text { Participação do } \\
\text { pai nos cuidados } \\
\text { com o filho }\end{array}$ & $\begin{array}{c}\text { Participação do pai } \\
\text { nas atividades } \\
\text { escolares, culturais } \\
\text { e de lazer do filho }\end{array}$ \\
\hline \multicolumn{7}{|c|}{$\begin{array}{l}\text { Problemas de } \\
\text { comportamento } \\
\text { Pais }\end{array}$} \\
\hline HIP. & $-0,251 *$ & $-0,258 * *$ & $-0,288 * *$ & $-0,303^{*}$ & $-0,271 * *$ & $-0,306^{* *}$ \\
\hline CPI. & $-0,200 *$ & ns & ns & ns & ns & ns \\
\hline CPE. & $-0,319 *$ & ns & $-0,226^{*}$ & $-0,295 * *$ & $-0,249 *$ & $-0,231 *$ \\
\hline CPT. & $-0,303^{*}$ & $0,228^{*}$ & $-0,257^{*}$ & $-0,293 * *$ & $-0,267 * *$ & $-0,268 * *$ \\
\hline \multicolumn{7}{|l|}{ Mães } \\
\hline HIP. & $-0,218^{*}$ & ns & ns & $-0,199^{*}$ & ns & ns \\
\hline CPE. & $-0,324 * *$ & $\mathrm{~ns}$ & ns & $-0,255^{*}$ & $-0,222 *$ & $-0,217 *$ \\
\hline CPT. & $-0,285 * *$ & ns & ns & $-0,220 *$ & $-0,198^{*}$ & $-0,208 *$ \\
\hline \multicolumn{7}{|c|}{ Habilidades Sociais } \\
\hline RHS - Pais. & $0,303 * *$ & $0,342 * *$ & $0,339 * *$ & $0,305 * *$ & $0,316^{* *}$ & $0,301 * *$ \\
\hline RHS-Mães. & $0,315^{* *}$ & $0,306^{* *}$ & $0,325^{* *}$ & $0,340 * *$ & $0,358 * *$ & $0,369 * *$ \\
\hline
\end{tabular}

Legenda: HIP: Hiperatividade; CPI: Comportamentos problemáticos internalizantes; CPE: Comportamentos problemáticos externalizantes; CPT: Comportamentos problemáticos total; RHS: Repertório de habilidades sociais.

$* p<0,05 ; * * p<0,01$ significativo;

ns $=$ não apresenta diferença estatisticamente significativa.

Quanto maiores os índices de hiperatividade e de problemas de comportamento (externalizantes e total) para as crianças (especialmente segundo a avaliação dos pais), menor a freqüência do envolvimento paterno nas três áreas investigadas (comunicação entre pai e filho, de participação do pai nos cuidados e nas atividades escolares, culturais e de lazer do filho), na opinião dos pais e das mães. Além disso, o repertório de habilidade sociais das crianças (segundo pais e mães) estava positivamente correlacionado com as três escalas indicadoras do envolvimento paterno, tanto na opinião dos pais quanto na das mães. Para os problemas de comportamento internalizantes, na percepção dos pais, apenas a escala de comunicação entre pai e filho (segundo os pais) apresentou uma correlação significativa e negativa. Não houve correlações significativas entre as opiniões das professoras quanto ao repertório social das crianças e ao envolvimento paterno.

\section{DISCUSSÃO}

O bom relacionamento pai-filho é preditor de uma relação afetuosa e positiva entre ambos e maximizador de diferentes aspectos do desenvolvimento infantil, como, por exemplo, o desenvolvimento social. A boa frequiência do envolvimento positivo dos pais, neste estudo, demonstra que muitos dos pais estão se comportando da maneira esperada pela nova paternidade, trabalhando junto com a esposa nos cuidados e na educação dos filhos (Brandth \& Kvande, 2002; Cia et al., 2006; Cia, Pereira, Del Prette \& Del Prette, 2006; Matta \& Knudson-Martin, 2006; Sayer, Gauthier \& Furstenberg, 2004; Tiedje, 2004).

Considerando-se os problemas de comportamento das crianças, notou-se que os três tipos de envolvimento paterno estavam negativamente correlacionadas com percepções da hiperatividade e dos problemas de comportamento do filho (externalizantes e total), segundo a avaliação de ambos os pais. Várias pesquisas mostram que os pais de crianças com mais problemas de comportamento têm estilos menos calorosos ou fazem pouco monitoramento e supervisão dos seus comportamentos (Anselmi, Piccinini, Barros \& Lopes, 2004; AtzabaPoria et al., 2004; Aunola \& Nurmi, 2005; Carlson, 2006; Coley et al., 2004; Davidov \& Grusec, 2006; 
Eisenberg et al., 2005; Fletcher, Steinberg \& Williams-Wheeler, 2006; Flouri, 2005; Formoso, Gonzáles, Barreira \& Dumka, 2007).

A baixa correlação entre os problemas de comportamento internalizantes e os indicadores de envolvimento paterno confirma que os problemas de comportamento internalizantes chamam menos a atenção do que os comportamentos que perturbam e alteram o meio, como os externalizantes. Tendo em vista que este estudo foi realizado com crianças que ingressavam no sistema escolar, vale comentar que os professores normalmente classificam essas crianças como tímidas, caladas ou "sem problemas", pois seus comportamentos não interferem no andamento das atividades em sala de aula e na atenção dos demais alunos (Atzaba-Poria et al., 2005; Caballo \& Simón, 2005; Cia et al., 2006).

Em contrapartida, as três medidas do envolvimento paterno foram positivamente correlacionadas com o repertório de habilidades sociais das crianças (BolsoniSilva \& Del Prette, 2002; Cia et al., 2006; Gomide, 2003; Pinheiro, Haase, Del Prette, Amarante \& Del Prette, 2006). É provável que, comparados com os pais menos envolvidos, os mais envolvidos estejam sendo modelos mais adequados de comportamentos socialmente habilidosos e propiciem às suas crianças uma maior frequiência de interações e estímulos sociais, favorecendo o desenvolvimento do seu repertório de habilidades sociais.

Segundo as professoras, pode ocorrer a nãocorrelação entre as medidas de envolvimento dos pais e a avaliação dos problemas de comportamento e do repertório de habilidades sociais das crianças, porque a maioria dessas medidas se referia ao contexto interno da família, e não ao contexto escolar.

É interessante ressaltar a utilização de dados de múltiplos informantes para verificar as correlações, por exemplo: (a) o envolvimento paterno foi avaliado pelos pais e mães e (b) a existência e a intensidade de problemas de comportamento e o repertório de habilidades sociais das crianças foram avaliados pelos pais, mães e professores, maximizando a confiança nos resultados (Cozby, 2002).

\section{CONSIDERAÇÕES FINAIS}

Este estudo levantou questões acerca do envolvimento paterno que podem influenciar $o$ desenvolvimento social dos filhos. Pôde-se verificar, de modo geral, que, embora as correlações não sejam tão fortes, quanto maior a frequiência de comunicação entre pai e filho e da participação do pai nos cuidados e nas atividades escolares, culturais e de lazer do filho, maior o repertório de habilidades sociais e menor o índice de hiperatividade e de problemas de comportamento externalizantes das crianças. Infere-se que programas educacionais com pais pouco envolvidos ou que apresentem um envolvimento inadequado com o filho, poderiam melhorar o envolvimento e por consequiência, resultar em um desenvolvimento mais saudável das crianças, como verificado em programas desenvolvidos em outros países (Duch, 2005; Fagan \& Stevenson, 2002).

Deve-se ressaltar que, por essas variáveis envolverem dados correlacionais, não podem ser estabelecidas conclusões sobre relação causal entre elas (Thompson, Diamond, McWilliam, Snyder \& Snyder, 2005). Além disso, os dados deste estudo baseiam-se no relato, podendo haver diferenças entre a percepção das pessoas envolvidas e o que realmente ocorre em seu diaa-dia. Sendo assim, estudos longitudinais e observacionais seriam indicados para monitorar a influência do envolvimento parental sobre o desenvolvimento social dos filhos ao longo do período do desenvolvimento infantil.

\section{REFERÊNCIAS}

Anselmi, L., Piccinini, C. A., Barros, F. C., \& Lopes, R. S. (2004). Psychosocial determinants of behavior problems in Brazilian preschool children. Journal of Child Psychology and Psychiatry, 45(4), 779-788.

Aunola, K., \& Nurmi, J. E. (2005). The role of parenting styles in children's problem behavior. Child Development, 76(6), 11441159.

Atzaba-Poria, N., Pike, A., \& Deater-Deckard, K.D. (2004). Do risk factors for problem behavior act in a cumulative manner? An examination of ethnic minority and majority children through an ecological perspective. Journal of Child Psychology and Psychiatry, 45(4), 707-718.

Bandeira, M., Del Prette, Z. A. P., Del Prette, A., \& Magalhães, T. (no prelo). Escala de avaliação das habilidades sociais de estudantes do ensino fundamental. SSRS-BR: Validação transcultural para o Brasil.

Bandura, A. (1986). Social foundations of thought and action: A social cognitive theory. Engelwood Cliffs, NJ: Prentice Hall.

Bolsoni-Silva, A. T., \& Del Prette, A. (2002). O que os pais falam sobre suas habilidades sociais e de seus filhos? Argumento, 3(7), 71-86.

Bongers, H. L., Koot, H. M., Ende, J. V. D., \& Verhulst, F. C. (2004). Developmental trajectories of externalizing behaviors in childhood and adolescence. Child Development, 75(5), 15231537.

Brandth, B., \& Kvande, E. (2002). Reflexive fathers: Negotiating parental leave and working life. Gender, Work and Organization, 9(2), 186-203.

Bronfenbrenner, U. (1999). Environments in developmental perspective: Theoretical and operational models. In S. L. Friedman \& T. D. Wachs (Orgs.), Measuring environment across 
the lifespan: Emerging methods and concepts. Washington, DC: American Psychological Association.

Caballo, V. E., \& Simón, M. A. (2005). Manual de psicologia clínica e do adolescente - Transtornos específicos. São Paulo: Santos.

Cabrera, N. J., Tames-LeMonda, C., Bradley, R. H., Hofferth, S., \& Lamb, M. E. (2000). Fatherhood in the twenty first century. Child Development, 71(1), 127-136.

Carlson, M. J. (2006). Family structure, father involvement, and adolescence behavioral outcomes. Journal of Marriage and Family, 68, 137-154.

Cia, F., D’Affonseca, S. M., \& Barham, E. J. (2004). A relação entre envolvimento paterno e desempenho acadêmico dos filhos. Paidéia: Cadernos de Psicologia e Educação, 14(29), 277-286.

Cia, F., Pamplin, R. C. O., \& Del Prette, Z. A. P. (2006). Comunicação e participação pais-filhos: correlação com habilidades sociais e problemas de comportamento dos filhos. Paidéia: Cadernos de Psicologia e Educação, 6(35), 395-406.

Cia, F., Pereira, C. S., Del Prette, Z. A. P., \& Del Prette, A. (2006). Habilidades sociais parentais e o relacionamento entre pais e filhos. Psicologia em Estudo, 11(1), 73-81.

Coley, R. L., Morris, J. E., \& Hernandez, D. (2004). Out-of-school care and problem behavior trajectories among low-income adolescents: Individual, family, and neighborhood characteristics as added risks. Child Development, 73(3), 948-965.

Cozby, P. C. (2002). Métodos de pesquisa em ciências do comportamento. São Paulo: Atlas.

Critério Brasil (2006). Associação brasileira de empresas de pesquisa. Recuperado em 05 de fevereiro, de 2007 em http://www.abep.org

Davidov, M., \& Grusec, J. E. (2006). Untangling the links of parental responsiveness to distress and warmth to child outcomes. Child Development, 77(1), 44-58.

Del Prette, Z. A. P., \& Del Prette, A. (2005). Psicologia das habilidades sociais na infância: Teoria e Prática. Petrópolis: Vozes.

Dessen, M. A., \& Costa, A. L. (2005). A ciência do desenvolvimento humano. Porto Alegre: ARTMED.

Duch, H. (2005). Redefining parent involvement in Head Start: A two-generation approach. Early Child Development and Care, 175(1), 23-35.

Dunn, J., Cheng, H., O’Connor, T. G., \& Bridges, L. (2004). Children's perspectives on their relationships with their nonresident fathers: Influences, outcomes and implications. Journal of Child Psychology and Psychiatry, 45(3), 553-566.

Eisenberg, N., Zhou, Q., Spinrad, T. L., Valiente, C., Fabes, R. A., \& Liew, J. (2005). Relations among positive parenting, children's effortful control, and externalizing problems: A three-wave longitudinal study. Child Development, 76(5), 1055-1071.

Fagan, J., \& Stevenson, H. C. (2002). An experimental study of an empowerment - based intervention for African American fathers. Family Relations, 51(3), 191-198.

Fletcher, A. C., Steinberg, L., \& Williams-Wheeler, M. (2006). Parental influences on adolescent problem behavior revisiting Stattin and Kerr. Child Development, 75(3), 781-796.
Flouri, E. (2005). Father's involvement and psychological adjustment in Indian and White British secondary school age children. Child and Adolescent Mental Health, 10(1), 32-40.

Formoso, D., Gonzáles, N. A., Barreira, M., \& Dumka, L. E. (2007). Interparental relations, maternal employment, and fathering in Mexican American Families. Journal of Marriage and Family, 69(1), 123-138

Gomide, P. I. C. (2003). Estilos parentais e comportamento anti-social. Em A. Del Prette \& Z. A. P. Del Prette (Orgs.), Habilidades sociais, desenvolvimento e aprendizagem (pp. 21-60). Campinas: Alínea.

Gresham, F. M., \& Elliott, S. N. (1990). Social skills rating system. Circle Pines, MN: American Guidance Service, Inc.

Marturano, E. M. (2004). Fatores de risco e proteção no desenvolvimento sócio-emocional de crianças com dificuldades de aprendizagem. Em E. G. Mendes, M. A. Almeida \& L. C. A. Williams (Orgs.), Avanços recentes em educação especial (pp.159-165). São Carlos: EDUFSCar.

Matta, D. S., \& Knudson-Martin, C. (2006). Father responsivity: Couple processes and the coconstruction of fatherhood. Family Process, 45(1), 19-37.

Oliveira, E. A., Marin, A. H., Pires, F. B., Frizzo, G. B., Ravanello, T., \& Rossato, C. (2002). Estilos parentais autoritário e democráticorecíproco intergeracionais, conflito conjugal e comportamento de externalização e internalização. Psicologia: Reflexão e Crítica, 15(1), 1-11.

Pinheiro, M. I. S., Haase, V. G., Del Prette, A., Amarante, C. L. D., \& Del Prette, Z. A. P. (2006). Treinamento de habilidades sociais educativas para pais de crianças com problemas de comportamento. Psicologia: Reflexão e Crítica, 19(3), 1-15.

Rocha, M. M., \& Brandão, M. Z. S. (2001). A importância do autoconhecimento dos pais na análise e modificação de suas interações com os filhos. Em M. Delitti (Org.), Sobre comportamento e cognição (pp. 133-141). Santo André: Esetec.

Sayer, L. C., Gauthier, A. H., \& Furstenberg, F. F. (2004). Educational differences in parents' time with children: Cross-national variations. Journal of Marriage and Family, 66, 1152-1169.

Thompson, B., Diamond, K. E., McWilliam, R., Snyder, P., \& Snyder, S. W. (2005). Evaluating the quality of evidence from correlational research for evidence-based practice. Exceptional Children, 71(2), 181-194.

Tiedje, L. B. (2004). Process of change in work/home incompatibilities employed mothers. Journal of Social Issues, 60(4), 787-800

Weber, L. N. D., Prado, P. M., Viezzer, A. P., \& Brandenburg, O. J. (2004). Identificação de estilos parentais: o ponto de vista dos pais e dos filhos. Psicologia: Reflexão e Crítica, 17(3), 323-331.

Recebido em 17/05/2007 Aceito em 17/10/2007

Endereço para correspondência : Fabiana Cia, Rodovia Washington Luís, Km 235, caixa postal: 676, Centro de Educação e Ciências Humanas, Departamento de Psicologia, Labor, CEP:13565905, São Carlos-SP, Brasil. E-mail: fabianacia@hotmail.com 